\title{
BMJ Open Optimisation and validation of a remote monitoring system (Onco-TreC) for home-based management of oral anticancer therapies: an Italian multicentre feasibility study
}

\author{
Alessandro Passardi, ${ }^{1}$ Mimma Rizzo, ${ }^{2}$ Francesca Maines, ${ }^{2}$ Carlo Tondini, ${ }^{3}$ \\ Alberto Zambelli, ${ }^{3}$ Roberto Vespignani, ${ }^{4}$ Daniele Andreis, ${ }^{5}$ Ilaria Massa, ${ }^{5}$ \\ Marco Dianti, ${ }^{6}$ Stefano Forti, ${ }^{6}$ Enrico Maria Piras, ${ }^{6}$ Claudio Eccher ${ }^{6}$
}

To cite: Passardi A, Rizzo M, Maines $\mathrm{F}$, et al. Optimisation and validation of a remote monitoring system (Onco-TreC) for home-based management of oral anticancer therapies: an Italian multicentre feasibility study. BMJ Open 2017;7:e014617. doi:10.1136/ bmjopen-2016-014617

- Prepublication history and additional material are available. To view these files please visit the journal online (http://dx.doi. org/10.1136/bmjopen-2016014617).

Received 12 0ctober 2016 Revised 24 March 2017 Accepted 5 April 2017

CrossMark

For numbered affiliations see end of article.

Correspondence to

Dr Daniele Andreis;

daniele.andreis@irst.emr.it

\section{ABSTRACT}

Introduction Despite the growing number of oral agents available for cancer treatment, their efficacy may be reduced due to the lack of adherence, inappropriate adverse event self-management and arbitrary dose adjustment. The management of anticancer therapies could exponentially benefit from the introduction of mobile health technologies in a highly integrated electronic oncology system.

Methods and analysis We plan to customise and finetune an existing monitoring TreC platform used in different chronic diseases in the oncology setting. This project follows a multistep approach with two major purposes: 1. participatory design techniques driven by Health Literacy and Patient Reported Outcomes principles in order to adapt the system to the oncology setting involving patients and healthcare providers; 2 . a prospective training-validation, interventional, non-pharmacological, multicentre study on a series of consecutive patients with cancer ( 20 and 60 patients in the training and validation steps, respectively) in order to assess system capability, usability and acceptability. The novel Onco-TreC 2.0 is expected to contribute to improving the adherence and safety of cancer care, promoting patient empowerment and patient-doctor communication.

Ethics and dissemination Ethical approval was obtained from the Independent Ethics Committees of the participating institutions (CEIIAV protocol Number 2549/2015; reference Number 1315-PU). Informed consent will be obtained from all study participants. Findings will be disseminated through peer-reviewed journals, conferences and event presentations.

Trial registration number ClinicalTrials.gov (NCT02921724); (Pre-results). Other study ID Number: IRST100.18.

\section{INTRODUCTION}

The use of oral chemotherapy or targeted therapy is growing in cancer care, leading to a major shift in the management of patients
Strengths and limitations of this study

- This multicentre study will be the first to have extended the TreC-platform-based monitoring system to the oncology setting (Onco-TreC).

- The Onco-TreC project is part of the European Innovation Partnership for Active and Healthy Ageing aiming to integrate mHealth tools in oncology.

- As this is a feasibility trial, it will not allow for definitive conclusions on the efficacy of the OncoTreC system.

- The restricted number of oral anticancer agents selected for this study might limit extrapolation of findings.

from directly observed intravenous therapy to self-administered home-based oral therapy, raising adherence and safety issues. ${ }^{1-5}$ Oral anticancer agents may produce side-effects which the patients will mostly self-manage from home. Although several studies showed that only $30 \%-50 \%$ of patients comply with drug prescriptions, a total of $16 \%-100 \%$ of patients adhere to oral anticancer agents. ${ }^{6}$ Poor adherence leads to negative health outcomes, growing frequency of hospital admission, more complex medication plans and higher use of healthcare resources.

The safety of home-based treatment is usually managed through different strategies: frequent medical visits, information booklets, patient-completed diaries and healthcare provider-patient phone contacts. Patient empowerment plays a key role in the self-management of therapies. ${ }^{89}$ The key points of empowerment include: information and communication between healthcare providers and patients to increase awareness of their 
health condition; knowledge of therapeutic programs and major drug interactions; competence in effective therapy management.

Patient-focused interventions acknowledge patients as active participants in the process of securing appropriate, effective, safe and responsive healthcare. ${ }^{10}$ To this aim, it is mandatory to move in the direction of a participatory and informed medicine, driven by Health Literacy and Patient Reported Outcomes principles. ${ }^{11}$ It is essential to form an alliance with patients by promoting effective communication, especially when their disease becomes chronic and affects them with comorbidities leading to the use of several concomitant medications. ${ }^{12}$ There is evidence in the literature that good, patient-centred communication is associated with positive health outcomes. ${ }^{13}$ In this context, 2.0 web solutions such as telemedicine, mobile health devices and applications (apps) are useful to improve adherence to medication ${ }^{14}$ and to optimise shared management of oral agents between patient and healthcare providers. ${ }^{15-18}$

The Center for Communication and Information Technology of the Fondazione Bruno Kessler (FBK-ICT) of Trento has developed a non-patented monitoring system based on the TreC (CCC, Citizen Clinical Record) platform to deliver mobile health services in different medical contexts. ${ }^{19}$ The monitoring system was tested in three pilot studies involving patients with chronic type 1 or 2 diabetes, hypertension and youth asthma. ${ }^{20-22} \mathrm{~A}$ living laboratory approach in real-life setting was used to test different groups of patient empowerment and self-care and relevant interventions by doctors and nurses. A total of 43 patients were involved in a three-step evaluation process: technical testing with a small number of operators; qualitative evaluations based on a small sample of patients; validation in clinical trial. These studies showed that the system was well accepted by patients and considered useful by physicians. Patients appreciated the closer interaction with healthcare providers and considered the system a convenient way to reduce direct access to hospital by raising their perception of safety. Given the similar characteristics of the patients studied in other chronic conditions at home and those treated with oral anticancer agents, results of previous studies provided a rationale for developing the system in an oncology setting. ${ }^{23}$ This clinical protocol describes the experimental design for answering our research question and explain how the data were collected and analysed during the project. This study will be jointly conducted at three Italian cancer care and research units (RUs): Istituto Scientifico Romagnolo per lo Studio e la Cura dei Tumori (IRST) IRCCS in Meldola, Forlì and Cesena (RU1); Oncology Unit of the Azienda Provinciale per i Servizi Sanitari (APSS) in Trento (RU2); Oncology Unit of the Azienda Socio-Sanitaria Territoriale (ASST) Papa Giovanni XXIII in Bergamo (RU3). The Information Technology approach promoted by FBK-ICT will serve as the communication link among RUs.

At RU1, different healthcare providers (eg, physicians, nurses, pharmacists, psychologists) closely cooperate to improve healthcare system processes. The patient working group 'CON-TATTO' regularly gives them an insight into the views of patients and their families. RU1 has implemented a multidisciplinary and multidimensional approach of therapeutic patient education in order to minimise toxicity and improve benefits of anticancer agents. Sixty-nine patients and caregivers prospectively assessed through preintervention and postintervention Beliefs about Medicines Questionnaire showed that this approach could raise their awareness of drugs in the management of their disease (data not published). RU1 is also the main contributor to the oncological Electronic Health Record (EHR) developed by the software LOG80, a comprehensive system which fosters patient safety, care quality and research. LOG80 supports therapy management during prescription, preparation and administration of antiblastic agents, as interoperable with other software and devices. ${ }^{24}$

At RU2, patients are informed by dedicated nurses about therapy and side-effects management. RU2, in collaboration with FBK-ICT, developed an information system (OncoSys) for the management of patients with cancer representing the backbone of Trentino Oncology Network. ${ }^{25}$

RU3 has been using a customised EHR for the management of patients with cancer based on the platform Galileo Electronic Health. RU3 recently launched a programme for the full-integration and free-interrogation of available patient databases created in the hospital from different sources. This programme relies on a web-based, open-source solution adopted with the aim to provide healthcare providers with an efficient and automated electronic data-warehouse. RU3 heads this programme, based on the I2B2 (Informatics for Integrating Biology and the Bedside) platform, which enables clinical researchers to use existing patient data for clinical management and research. RU3 is part of the I2B2 International Network for the development of ultimate IT solutions for cancer healthcare management.

\section{AIMS AND OBJECTIVES}

The aim of the study is to customise and fine-tune a 2.0 mobile health system in order to optimise patient-doctor communication, home-based management and remote monitoring of oral anticancer agents.

The objectives of the study are as follows:

1. To redesign an existing system in an oncology setting (Onco-TreC) by involving patients and healthcare providers through participatory design techniques.

2. To assess capability of Onco-TreC to record critical data of medication adherence and safety, promoting patient-doctor communication and improving the shared management of oral anticancer agents.

3. To assess usability and acceptability of Onco-TreC by patients and healthcare providers, monitoring patient-perceived levels of quality of life, anxiety, 
quality of care and patient-doctor communication over time.

\section{METHODS AND ANALYSIS}

Both qualitative and quantitative research approaches will be used in this project, following two sequential phases:

- participatory design techniques in order to adapt the system to the oncology setting (objective 1);

- a prospective study in order to assess Onco-TreC capability, usability and acceptability (objectives 2 and 3).

\section{PARTICIPATORY RESEARCH Approach}

Redesigning an existing 2.0 system in an oncology setting will require the active involvement of patients and healthcare providers. This is an innovative change from a user-centred design process ('for' users) to a shared process ('with' users) through participatory design techniques (eg, focus group sessions, joint reviews). ${ }^{26}$ Face-to-face participatory design sessions will be conducted at baseline in each RU, involving healthcare providers and patient representatives. Results of these local sessions will be shared among the RUs by face-toface central sessions. Additional face-to-face and on-line participatory sessions will be planned during the study. Results of such sessions will be uploaded onto the system by FBK-ICT. A shared revision of Common Terminology Criteria for Adverse Events (CTCAE) Version $4.03^{27}$ based on Health Literacy and Patient Reported Outcomes principles will be included in the system to facilitate patient self-reporting of adverse events and treatment evaluation.

Onco-TreC will be composed of two tools aimed at supporting patient self-care and healthcare provider monitoring and intervention:
- Mobile diary app. This is an Android app which allows patients to record parameters related to their health state (eg, medications; blood pressure, weight, body temperature; side-effects or other symptoms; personal notes) through a mobile device (figures 1 and 2). Data are confidential and stored in a central database, ensuring sensitive data management compliance with the Personal Data Protection Code (D.Lgs. 196/03) and the European Data Protection Regulation (Directive 95/46/EC). Data can be selectively made available in real time to healthcare providers through a web dashboard on the TreC server or through a tablet app. At each data input, the app provides the patient with tips for the promotion of critical event self-management. The app sends the user's data to the secure server where a web service with a rule-based alarm system analyses the incoming parameters and notifies healthcare providers of any critical condition via email.

- Web dashboard (figure 3). Through the secure access web portal, oncologists can check patient data, monitor side-effects and adherence to prescriptions, answer patient's personal messages or write to the patient. In case of critical health condition or alarming lack of adherence, oncologists can contact the patients, collect supplementary information and take any necessary action to solve the problem.

Patient-doctor communication is supported by two modules: the 'Alarms + tips' module, for remote monitoring and self-care and the 'Secure messaging' module, for asynchronous communication between patients and healthcare providers. Two user guides (one for patients and one for healthcare providers) will be created to provide stepwise instructions on how to use Onco-TreC.

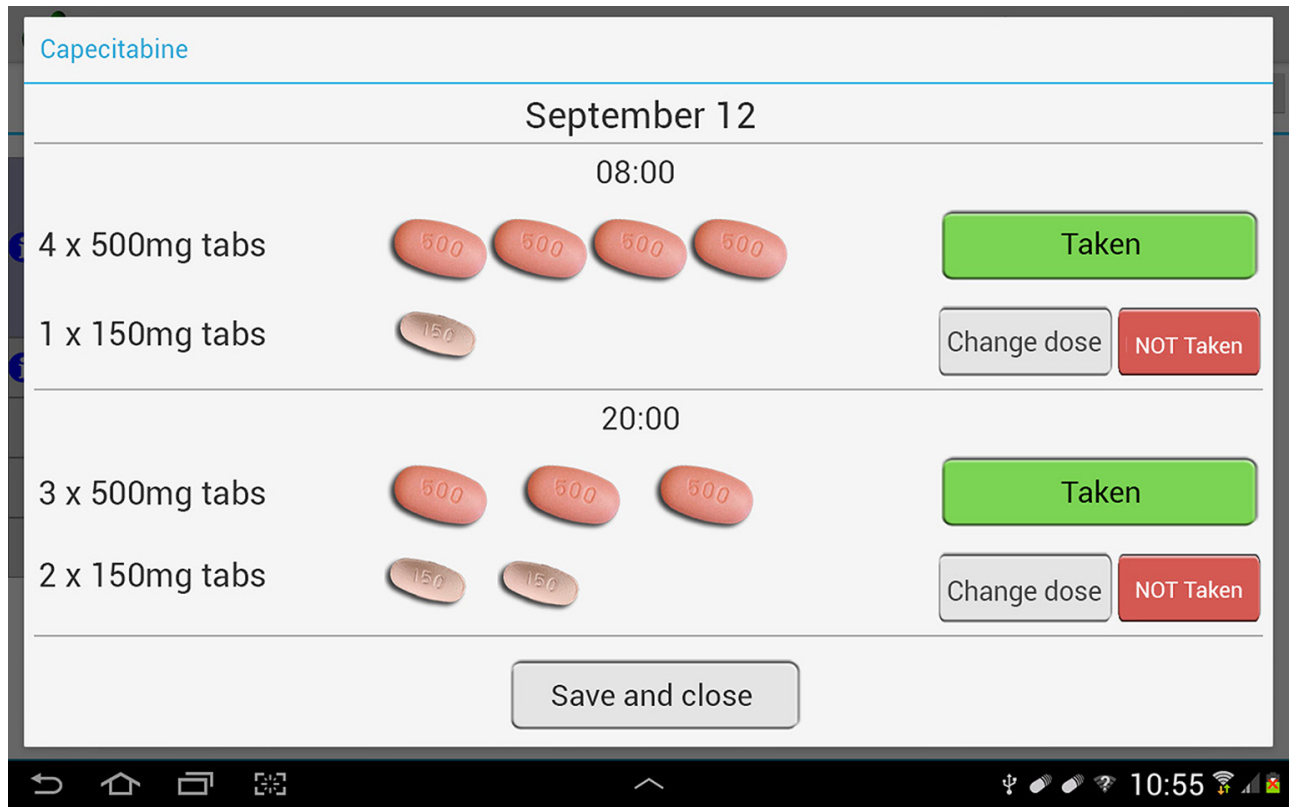

Figure 1 Screenshot of the diary showing the window that allows patients to register the tablets taken daily. 


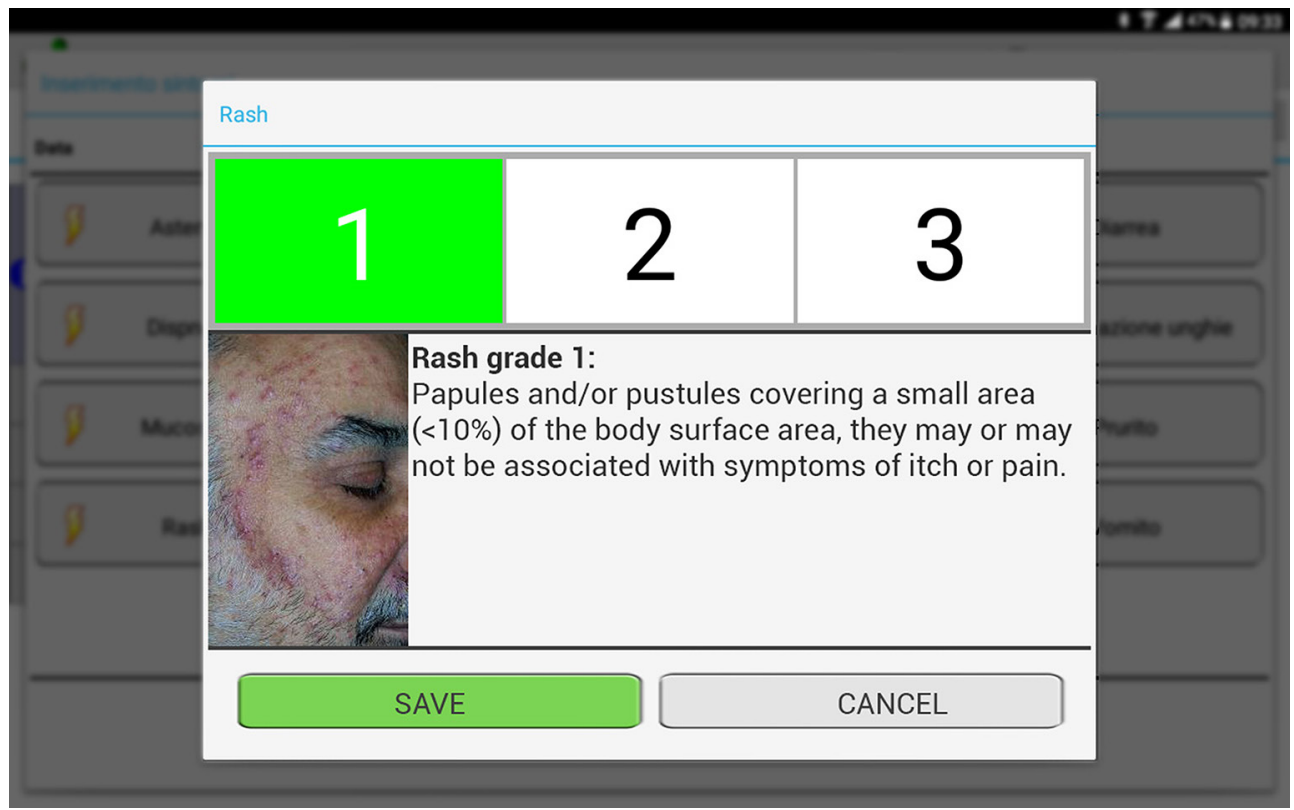

Figure 2 Screenshot of the diary showing the window that allows patients to register the onset and intensity of therapy-related rash. The app helps patients to determine the toxicity grade by displaying explanatory texts and pictures.

A dedicated phone number will be activated for technical support during the project.

\section{Participating centres}

This project will be jointly conducted at RU1 in Meldola, Forlì and Cesena, RU2 in Trento and RU3 in Bergamo. The multidisciplinary team and the Information Technology approach promoted by FBK-ICT will guarantee synergic activities among RUs and mutual access to research results.

\section{PROSPECTIVE STUDY}

\section{Study population}

The system will be assessed by performing a prospective training-validation, interventional, non-pharmacological, multicentre study on a series of consecutive cancer patients accessing three cancer centres in different Italian regions.

A total of 80 evaluable patients will be considered: 20 patients in the training step and 60 patients in the

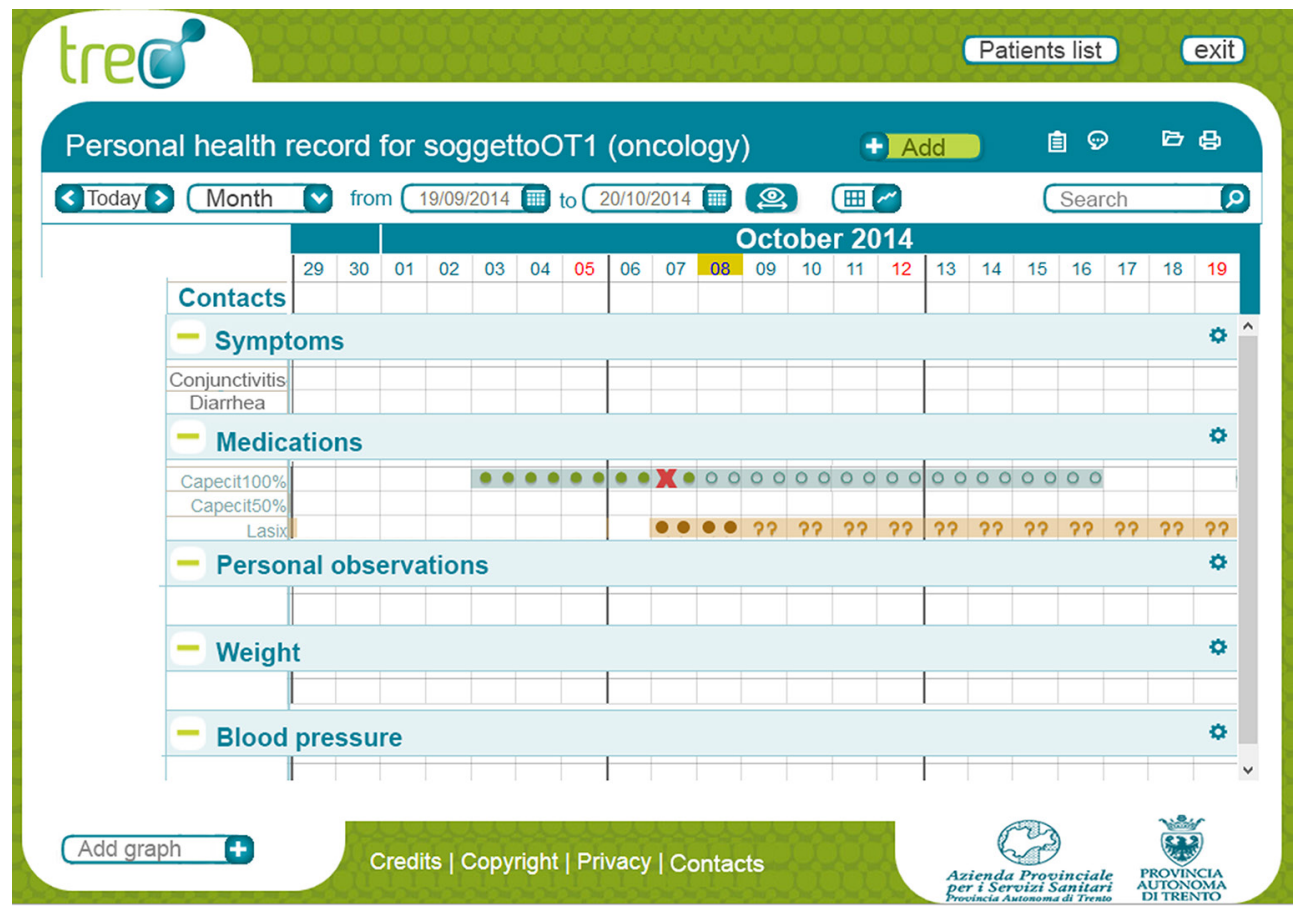

Figure 3 Screenshot of the oncology dashboard via the web browser. Charts show patient health data, for example, weight, symptoms, personal observations and therapy adherence data. 
validation step. Eligible patients must meet the following criteria:

- adult $\geq 18$ years old, either sex;

- ECOG-Performance Status $\leq 1$;

- life expectancy >12 weeks according to clinical judgement;

- candidate for treatment with an oral agent as monotherapy, for example, capecitabine or sunitinib (adjuvant and advanced settings allowed);

- good understanding of the Italian language;

- ability to follow study procedures and manage mobile devices after a basic training course, at the investigator's discretion;

written informed consent.

Patients receiving intravenous anticancer treatment will be excluded, in order to reduce potential confounding in evaluating the system.

\section{Study procedures}

To assess Onco-TreC capability, 20 eligible patients will enter the study in the training step. Healthcare providers will supply the device to the patients with complete information on mobile app use and functions. Patients' data (eg, patient demographics; medical history; prior and concomitant medications; details of physical examinations and vital signs) will be recorded at baseline and will be updated during the following medical visits. Information on modifications of concomitant diseases and treatments, adverse events according to CTCAE Version 4.03, drug accountability and adherence checks will also be collected. Patients will be required to manually enter data into the system at least once a day. Patients will be seen at the centres every 6 weeks. Patients enrolled in the training step will remain under observation for a minimum of 6 weeks and a maximum of 12 weeks, except when a change of therapy occurs (due to either disease progression, or unacceptable toxicity, or medical/patient decision). At the end of the training step, results will be examined and the system will be fine-tuned.

In the validation step, a further 60 patients will be recruited with the same characteristics as those of the training step to better assess adherence, prevention of serious home complications, dose reduction or treatment interruption and emergency room accesses. Patients enrolled in the validation step will remain under observation for a maximum of 24 weeks.

Usability and acceptability of Onco-TreC by patients will be assessed through Functional Assessment of Cancer Therapy-General (FACT-G) ${ }^{28}$ and Hospital Anxiety and Depression Scale (HADS) ${ }^{29}$ questionnaires, electronically self-administered at baseline, during treatment and at the at the end of observation (EoO). Other three self-administered questionnaires will be filled by the patients: Q-pre and Q-post at baseline and at $\mathrm{EoO}$, respectively; Italian version of the System Usability Scale (SUS) ${ }^{30}$ at EoO.

All patients enrolled in the training step and the first 20 patients in the validation step will undergo semi-structured interviews by FBK-ICT sociologists at EoO. These interviews will be held at the patient's home or at their medical centre and will focus on care practice and the use of the new technology. FBK-ICT sociologists will also conduct semi-structured interviews with the oncologists to evaluate the impact of the technology on their workload, patient-doctor communication, patient adherence and safety issues.

\section{Data management and analysis}

The first customised version of the system may not fully meet information needs. During the study, the participatory design techniques and the multistep approach will allow us to identify and solve emerging problems in terms of system capability and participant usability and acceptability.

Onco-TreC app will communicate with a back-end service to store data on a central server. Researchers will be able to evaluate capability data through a web-based dashboard. Data entered into the system by the patient at home will be compared with those assessed by the oncologist during the visit, in particular: patient-entered toxicities or number of pills will be compared with those directly reported to the doctor or compared with the number of the residual pills returned during the hospital visit, respectively. Data will be registered in electronic case report forms implemented using a relational database management system and a graphic user interface.

Patient perception about Onco-TreC will be assessed by subdividing FACT-B and HADS scores into different subscales and by analysing them using the Wilcoxon rank-sum test. We will translate into Italian (by forward-translation and back-translation) an internationally validated SUS to investigate the system usability. A review of the literature did not reveal any existing validated questionnaires to assess other specific areas of our project. Two specific questionnaires (Q-pre and Q-post) were also developed to evaluate patient expectations about the system, system acceptability, quality of care and patient-doctor communication. The semi-structured interviews will be audio recorded, transcribed and assessed by the template analysis, a structured technique for the evaluation of qualitative data.

A formal sample size calculation for the prospective study was not performed due to the lack of preliminary data in an oncology setting and results from controlled before-and-after studies. Descriptive statistics will be used for data analysis due to the exploratory intent of the study. An interim analysis is planned at the end of the training step and at EoO of the first 20 patients enrolled in the validation step. Once these preliminary data are available, a statistical approach based on clinical experience to estimate the system capability can be defined.

\section{ETHICS AND DISSEMINATION}

This Italian multicentre feasibility study, approved by the Independent Ethics Committees of the participating institutions (CEIIAV protocol Number 2549/2015; reference 
Number 1315-PU), will be conducted in accordance with the Declaration of Helsinki and the Good Clinical Practice guidelines.

The TreC-platform-based monitoring system has been tested in different chronic diseases but has never been extended to the oncology setting. The innovative tool for home-based management of oral agents has been shown to improve quality and safety of care, promote adherence to oral agents and reduce risk of drug interactions, serious complications, improper dose self-adjustment and emergency room access. The extension of this project to the Italian National Health Service will have a twofold benefit: to promote patient self-empowerment and patientdoctor communication and to ensure the sustainability and efficiency of the healthcare system.

Unlike most electronic health tools implemented by local health services, Onco-TreC will be language editable, easily adaptable to agents with several toxicities and interoperable with different EHRs and hospital workflows. Onco-TreC project is part of the European Innovation Partnership for Active and Healthy Ageing as Good Practice of the Emilia Romagna Region, in particular in the A1 Synergy Group on 'Information Technology and Adherence in Ageing Population with Chronic Diseases and Polypharmacy'.

Our findings will provide reliable post-approval data comparable with adherence and safety results from pivotal and other relevant studies on specific oral anticancer agents. It thus holds great promise to substantially impact and benefit a large audience. The results will be disseminated through peer-reviewed journals, conferences and event presentations.

\section{Author affiliations}

${ }^{1}$ Department of Medical Oncology, Istituto Scientifico Romagnolo per lo Studio e la Cura dei Tumori (IRST) IRCCS, Meldola, Italy

${ }^{2}$ Department of Medical Oncology, Azienda Provinciale per i Servizi Sanitari, Trento, Italy

${ }^{3}$ Department of Medical Oncology, Azienda Socio-Sanitaria Territoriale Papa Giovanni XXIII, Bergamo, Italy

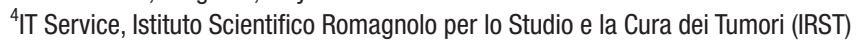
IRCCS, Meldola, Italy

${ }^{5}$ Biostatistics and Clinical Trials Unit, Istituto Scientifico Romagnolo per lo Studio e la Cura dei Tumori (IRST) IRCCS, Meldola, Italy

${ }^{6}$ Center for Information and Communication Technology, eHealth Unit, Fondazione Bruno Kessler, Trento, Italy

Acknowledgements The authors would like to thank Veronica Zanoni and Cristiano Verna for editorial assistance.

Contributors AP, MR, FM, CT, AZ, RV, DA, IM, MD, SF, EMP and CE designed the study. MD, SF, EMP and CE provided study materials. CE, AP and DA prepared the manuscript. All authors were involved in reviewing and commenting on the manuscript and approved the final paper.

Funding There are no funders to report for this study. The project was submitted in 2016 to the Italian Ministry of Health Call for Targeted Research Grant (Project Code: RF-2016-02362926).

\section{Patient consent Obtained.}

Ethics approval CEIIAV protocol Number 2549/2015; reference Number 1315-PU.

Provenance and peer review Not commissioned; externally peer reviewed.

Open Access This is an Open Access article distributed in accordance with the Creative Commons Attribution Non Commercial (CC BY-NC 4.0) license, which permits others to distribute, remix, adapt, build upon this work non-commercially, and license their derivative works on different terms, provided the original work is properly cited and the use is non-commercial. See: http://creativecommons.org/ licenses/by-nc/4.0/

(c) Article author(s) (or their employer(s) unless otherwise stated in the text of the article) 2017. All rights reserved. No commercial use is permitted unless otherwise expressly granted.

\section{REFERENCES}

1. Osterberg L, Blaschke T. Adherence to medication. N Engl J Med 2005;353:487-97.

2. Brown MT, Bussell JK. Medication adherence: who cares? Mayo Clin Proc 2011;86:304-14.

3. Huang WC, Chen CY, Lin SJ, et al. Medication adherence to oral anticancer drugs: systematic review. Expert Rev Anticancer Ther 2016;16:423-32.

4. Bergsbaken JJ, Eickhoff JC, Buss BA, et al. Assessment of adherence with oral anticancer agents in oncology clinical trials: a systematic review. J Oncol Pharm Pract 2016:22:105-13.

5. Greer JA, Amoyal N, Nisotel L, et al. A systematic review of adherence to oral antineoplastic therapies. Oncologist 2016;21:354-76.

6. Fenerty SD, West C, Davis SA, et al. The effect of reminder systems on patients' adherence to treatment. Patient Prefer Adherence 2012;6:127-35.

7. Vervloet M, van Dijk L, Santen-Reestman J, et al. SMS reminders improve adherence to oral medication in type 2 diabetes patients who are real time electronically monitored. Int J Med Inform 2012;81:594-604.

8. Oakley C, Lennan E, Roe H, et al. Safe practice and nursing care of patients receiving oral anti-cancer medicines: a position statement from UKONS. Ecancermedicalscience 2010;4:177.

9. Mathes T, Antoine SL, Pieper D, et al. Adherence enhancing interventions for oral anticancer agents: a systematic review. Cancer Treat Rev 2014:40:102-8.

10. Coulter A, Ellins J. Effectiveness of strategies for informing, educating, and involving patients. BMJ 2007;335:24-7.

11. Dewalt DA, Berkman ND, Sheridan S, et al. Literacy and health outcomes: a systematic review of the literature. J Gen Intern Med 2004;19:1228-39.

12. Lees J, Chan A. Polypharmacy in elderly patients with Cancer: clinical implications and management. Lancet Oncol 2011;12:1249-57.

13. Stewart MA. Effective physician-patient communication and health outcomes: a review. Cmaj 1995;152:1423-33.

14. Anglada-Martínez $\mathrm{H}$, Martin-Conde M, Rovira-Illamola M, et al. Feasibility and preliminary outcomes of a web and SmartphoneBased medication Self-Management Platform for Chronically III Patients. J Med Syst 2016;40:99.

15. Weaver A, Young AM, Rowntree J, et al. Application of mobile phone technology for managing chemotherapy-associated side-effects. Ann Oncol 2007;18:1887-92

16. Paré G, Moqadem K, Pineau G, et al. Clinical effects of home telemonitoring in the context of diabetes, asthma, heart failure and hypertension: a systematic review. J Med Internet Res 2010;12:e21.

17. Polisena J, Tran K, Cimon K, et al. Home telehealth for chronic obstructive pulmonary disease: a systematic review and metaanalysis. J Telemed Telecare 2010;16:120-7.

18. Polisena J, Tran K, Cimon K, et al. Home telemonitoring for congestive heart failure: a systematic review and meta-analysis. J Telemed Telecare 2010;16:68-76.

19. Eccher C, Piras EM, Stenico M. TreC - a REST-based regional PHR. Stud Health Technol Inform 2011;169:108-12.

20. Franceschi R, Cauvin V, Piras EM, et al. Trec diabetes: a new telemedicine system. In: Diabetes technology \& therapeutics, 2014 Presented at: ATTD 2014, 7th International Conference on Advanced Technologies \& Treatments for Diabetes; February 5-8, 2014; Wien.

21. Maria Piras E, Zanutto A. 'One day it will be you who tells us doctors what to do!'. Exploring the 'Personal'of PHR in paediatric diabetes management. Info Tech People 2014;27:421-39.

22. Eccher C, Botteri M, Ortolani D, et al. A mobile logbook to diagnose masked hypertension: a pilot application. Stud Health Technol Inform 2014;205:363-7.

23. Galligioni E, Piras EM, Galvagni M, et al. Integrating mHealth in Oncology: experience in the Province of Trento. J Med Internet Res 2015;17:e114.

24. Masini C, Nanni O, Antaridi S, et al. Automated preparation of chemotherapy: quality improvement and economic sustainability. Am J Health Syst Pharm 2014;71:579-85. 
25. Galligioni E, Berloffa F, Caffo O, et al. Development and daily use of an electronic oncological patient record for the total management of Cancer patients: 7 years' experience. Ann Oncol 2009;20:349-52.

26. Sanders EBN. From user-centered to participatory design approaches. Taylor \& Francis. London: Design and the social sciences, 2002.

27. CTCAE is widely accepted throughout the oncology community as the standard classification and severity grading scale for adverse events in cancer therapy clinical trials and other oncology settings The National Cancer Institute issued the CTCAE version 4.0 on May 29, 2009. An updated version (4.03) is now in use as of June 14, 2010. PDF of CTCAE v 4.03 can be downloaded at . http://evs.
nci.nih.gov/ftp1/CTCAE/CTCAE_4.03_2010-06-14_QuickReference 8.5x11.pdf (accessed 26 July, 2016).

28. Webster K, Cella D, Yost K. The Functional Assessment of Chronic illness therapy (FACIT) Measurement System: properties, applications, and interpretation. Health Qual Life Outcomes 2003;1:79.

29. Costantini M, Musso M, Viterbori P, et al. Detecting psychological distress in Cancer patients: validity of the Italian version of the Hospital anxiety and depression Scale. Support Care Cancer 1999;7:121-7.

30. Brooke J. SUS: A 'quick and dirty usability scale'. In: Jordan PW, Thomas B, Weerdmeester BA, Usability evaluation in industry. London, Taylor \& Francis, 1996:189-94. 